2 Wiesbeck GA, Weijers HG, Lesch OM, Glaser T, Toennes PJ, Boening J. Flupenthixol decanoate and relapse prevention in alcoholics: results from a placebo-controlled study. Alcohol Alcohol 2001; 36: 329-34.

3 Guardia J, Segura L, Gonzalvo B, Iglesias L, Roncero C, Cardús M, et al. A double-blind, placebo-controlled study of olanzapine in the treatment of alcohol-dependence disorder. Alcohol Clin Exp Res 2004; 28: 736-45.

4 Kishi T, Sevy S, Chekuri R, Correll CU. Antipsychotics for primary alcohol dependence: a systematic review and meta-analysis of placebo-controlled trials. J Clin Psychiatry 2013; 74: 642-54.

Jonathan Chick, Medical Director, Castle Craig Hospital, and Visiting Professor, School of Health and Social Care, Napier University, Edinburgh. Email: jonathan.chick@gmail.com; Colin Drummond, Professor of Addiction Psychiatry; jonathan.chick@gmail.com; Colin

doi: 10.1192/bjp.211.1.52a

population and how these may vary as a consequence of medical severity.

1 Chitty KM, Dobbins T, Dawson AH, Isbister GK, Buckley A. Relationship between prescribed psychotropic medications and co-ingested alcohol in intentional self-poisonings. Br J Psychiatry 2017; 210: 203-8.

2 Matsuyama T, Kitamura T, Kiyohara K, Hayashida S, Kawamura T, Iwami T, et al. Characteristics and outcomes of emergency patients with self-inflicted injuries: a report from ambulance records in Osaka City, Japan. Scand J Trauma Resusc Emerg Med 2016; 24: 68.

3 Lloyd B, Gao CX, Heilbronn C, Lubman DI. Self Harm and Mental Health Related Ambulance Attendances in Australia: 2013 Data. Turning Point, 2015.

Katrina Witt, Post-Doctoral Research Fellow, Turning Point, Eastern Health Clinical School, Monash University, Australia. Email: katrina.witt@monash.edu; Dan Lubman Professor of Addiction Studies and Services; Belinda Lloyd, Associate Professor of Professor of Addiction Studies and Services; Belinda Lloyd, Associate Professor of
Addiction Studies and Services; Karen Smith, Manager, Research and Evaluation and Adjunct Professor, Monash University, Australia

doi: 10.1192/bjp.211.1.53

\section{Co-consumption of alcohol and psychotropic medications in episodes of non-fatal self-poisoning attended by ambulance services in Victoria, Australia: evidence of potential modification by medical severity}

Chitty and colleagues' recent investigation ${ }^{1}$ into the association between psychotropic medication use and alcohol consumption during emergency department presentations for self-poisoning raises an interesting perspective on the putative role of psychopharmacology in reducing risky alcohol use among those at risk of self-harm and suicide.

Episodes of attempted suicide resulting in hospital presentation may underestimate the true extent of psychotropic medication and alcohol co-ingestion across the community, given recent findings suggesting that just over half of patients treated by ambulance paramedics following an episode of self-harm and/or attempted suicide are transported to hospital. ${ }^{2}$

Using data from our ongoing study of psychiatric presentations to ambulance services, ${ }^{3}$ we extracted information on all episodes of non-fatal self-poisoning in the state of Victoria, Australia, from January 2012 to December $2016(N=24726)$. In contrast to Chitty and colleagues, we found that, overall, use of psychotropic medications was associated with an increased, not decreased, risk of alcohol co-consumption in the self-poisoning episode (odds ratio $(\mathrm{OR})=1.35,95 \% \mathrm{CI} 1.28-1.42)$.

While anticonvulsants $(\mathrm{OR}=0.74,95 \%$ CI $0.65-0.84)$, antipsychotics $(\mathrm{OR}=0.81,95 \% \mathrm{CI} 0.75-0.86)$ and psychostimulants $(\mathrm{OR}=0.52,95 \%$ CI $0.32-0.85)$ were associated with a decreased risk of alcohol co-consumption, in contrast to Chitty and colleagues, we found that benzodiazepines $(\mathrm{OR}=1.60,95 \% \mathrm{CI}$ 1.52-1.69) were associated with an increased risk of alcohol co-consumption. Additionally, we found no significant association between antidepressant use and risk of alcohol co-consumption for these presentations $(\mathrm{OR}=1.04,95 \%$ CI $0.97-1.11)$.

Importantly, however, we found that medical severity may modify these associations. Specifically, most associations were reduced to non-significance when considering those not requiring hospital treatment following the self-poisoning episode: all psychotropic medication classes $(\mathrm{OR}=1.12,95 \%$ CI $0.76-1.65)$, anticonvulsants ( $\mathrm{OR}=0.39,95 \% \mathrm{CI} 0.09-1.80)$, antidepressants $(\mathrm{OR}=1.05,95 \%$ CI $0.63-1.77)$, antipsychotics $(\mathrm{OR}=0.81,95 \%$ CI $0.48-1.36)$, benzodiazepines $(\mathrm{OR}=1.40,95 \%$ CI $0.94-2.07)$ and psychostimulants (OR $=0.44,95 \%$ CI $0.02-9.21)$.

This highlights the importance of considering the breadth of services that people who engage in self-harm come into contact with, so as to provide a fuller picture of the treatment needs of this
Authors' reply: We thank Chick et al, and Witt et al, for their welcome responses to our article. ${ }^{1}$ We agree that both improving access and facilitating referral to alcohol services are essential strategies with regard to reducing deliberate self-poisoning that may be a product of harmful use of alcohol.

We share the concerns of Chick and colleagues - it is dangerous to make causal assertions from cross-sectional data, especially if preliminary analyses and author interpretations are introduced into clinical practice from the abstract alone. We agree that people prescribed tricyclic antidepressants and typical antipsychotics are different from those on other drugs - that they are less likely to co-ingest alcohol during intentional self-poisoning is one such example. As highlighted by Chick et al, the underlying nature of this relationship (whether it is causal or correlated because of shared factors) has many possibilities, for which we presented three interpretative and non-mutually exclusive speculations. We agree with the further interpretation put forth in their letter - individuals with increased access to higher-toxicity medications may negate any perceived role of alcohol in the poisoning. Of course, this is only relevant in cases when alcohol is used as a tool to facilitate the self-harm (i.e. to 'numb fears') as opposed to the person being intoxicated before the desire to self-harm arises. It is noteworthy that a recent study found that over $70 \%$ of people interviewed after a suicide attempt that involved acute alcohol use reported they did not use alcohol to facilitate the action. ${ }^{2}$ However, we recognise that the methods of suicide attempts in this aforementioned small sample size study were heterogeneous and that self-poisoning is more likely to involve alcohol as a substance perceived to increase the toxicity of the poison or mask the taste of the co-ingested substances. We are currently conducting a study to investigate patient selfreported reasons for use of alcohol before and during deliberate self-poisoning, which will further shed light on this.

We are pleased that our analysis prompted Witt and colleagues to investigate a similar line of enquiry within their own cohort. The similarities between the data analysis conducted by Witt et al and our findings are notable - those prescribed antipsychotics, anticonvulsants and stimulants were less likely to co-ingest alcohol during a non-fatal self-poisoning.

Compared with the Japanese study cited by Witt et al, in which nearly half of individuals are not transported to hospital after suicide attempts or episodes of self-harm, our experience specifically for deliberate self-poisoning (via toxicology services and Poison Information Centres) tells us this is not the case in 